\title{
The Use of Medical Cannabis on Cancer in Thailand
}

\author{
Sirisopha Ekarattanawong ${ }^{1}$, Varissara Ketphan ${ }^{2}$, Yada Rojcharoenchai ${ }^{3}$ \\ ${ }^{1}$ Suankularbwittayalai Rangsit School, ${ }^{2}$ Triamudom Suksa School, ${ }^{3}$ Saipanya Rangsit School, Thailand \\ Corresponding Author: Sirisopha Ekarattanawong
}

\begin{abstract}
The objective of this paper is to indicate the beneficial utility of medical marijuana. Marijuana throughout history is known for its property to alternate consciousness. However, the medical utilization of marijuana or cannabis was dated as far back as 2900 BC, when it was used by Emperor Ru Hsi of Ancient Chinese. During the 19th century, marijuana was introduced to Western Medicine as a therapeutic drug, mostly known for its pain control properties. Marijuana by itself consists of more than 100 active components. In consideration of the amount of THC, tetrahydrocannabinol, a psychological chemical released by the glands of marijuana plants, CBD or cannabidiol, amongst the most prevalent ingredients in cannabis, is the least controversial extract extracted from the marijuana plants to be used. As of the year 2019, Thailand Narcotics Act legalized cannabis for medical use in Thailand. A study survey conducted by N.Z. shows that in just over a year, $20 \%$ of the patients report the use of cannabis for medical purposes regarding its benefits of neuropathic pain, chemotherapyinduced nausea and vomiting, Aids-related cachexia, intractable epilepsy, and palliative care conditions. Further clinical trials are conducted to further perceive the potential cannabis has on treating cancer. One of the two successful clinical trials that have been published proposes that cannabis may make it possible to treat brain cancer with few side effects.
\end{abstract}

Keywords: Cancer, Marijuana, Tetrahydrocannabinol (THC), Cannabidiol (CBD), Medical Usage, Cannabis in Thailand

\section{INTRODUCTION 1.1 The Causes of Cancer}

Cancer is a condition characterised by uncontrollable cell growth that spreads to other parts of the body. In the millions of cells that make up the human body, cancer can start almost anywhere. Through a process called cell division, human cells normally develop and multiply to generate new cells which are needed. Cells die as they become old or damaged, and new cells replace them. However, sometimes, this ordered process can break down. It results in aberrant or damaged cells growing and multiplying when they shouldn't. ${ }^{[1]}$ Tumors, which are masses of tissues, can grow from these cells. Tumors may or may not be malignant. Noncancerous tumors are called benign tumors. The majority of benign tumors grow slowly and do not spread to other parts of the body. However, malignant tumors which are cancerous ones can spread throughout the body via the circulatory or the lymphatic system (metastasis). They grow rapidly, infiltrate and destroy normal tissues which are nearby. ${ }^{[2-3]}$

Everyone has a risk of developing cancer. Many cancers appear to arise out of nowhere. Since cancer is a complex multistep process with several elements influencing the likelihood of acquiring it, determining the single cause for most cancers is difficult. Nevertheless, certain risk factors are identified by scientists to increase the likelihood that one or more of the cells in a human body will become abnormal and lead to cancer. Radiation and other chemical carcinogens damage DNA and cause mutations. Carcinogens are 
chemical substances that have the potential to cause cancer. The risk of cancer increases with the amount of time spent in contact with a carcinogen. Carcinogens in tobacco smoke (including benzo( $a$ )pyrene, dimethylnitrosamine, and nickel compounds) are the most commonly diagnosed causes of cancer in humans. Some other chemicals such as asbestos, benzene, and formaldehyde can increase the risk of developing certain cancers. Other carcinogens, rather than causing mutations, encourage cancer progression through stimulating cell proliferation. These compounds are called tumor promoters because they cause accelerated cell division, which is necessary for the outgrowth of a proliferative cell population in the early stages of tumor development. Phosphorbol esters, which enhance cell proliferation by activating protein kinase $\mathrm{C}$, are an example of a tumor promoter. Other than that, hormones, specifically estrogens, play a role in the development of various human cancers as tumor promoters. Viruses, as well as chemicals and radiation, have been shown to cause cancer in both experimental animals and humans. Yet, other causes of cancer are not preventable, such as age and genetic disorders. $^{[4-5]}$

\subsection{Types of Cancer}

If cancer is classified by where it starts in the body, such as breast or lung cancer, there are approximately 200 different varieties. ${ }^{[6]}$

Another way to categorise cancers is based on the type of fluid or tissue in which they start. There are five major categories. These are the following:

- Sarcoma is a cancerous tumor that begins in the connective tissues of the body like the bones, muscles, cartilage, and blood vessels.

- Lymphoma is a malignant tumor or cell that develops in the lymphatic system's nodes and glands. They take responsibility for producing white blood cells to decontaminate blood fluids.
- Leukemia, frequently known as blood cancer, is a bone marrow malignancy that inhibits the bone marrow from generating typical red blood cells, white blood cells, and platelets.

- Myeloma is a cancer that develops in the bone marrow's plasma cells.

Furthermore, certain cancers are mixed kinds. ${ }^{[7]}$

\subsection{Characteristics of Cancer Pain}

Pain is a common symptom addressed by healthcare professionals in a palliative care team, and cancer is a prevalent clinical disease encountered in palliative care. Cancer pain is now recognised as a phenomenon rather than a symptom, condition, or mechanism. ${ }^{[8]}$ The majority of cancer pain is caused by the tumor pressing against bones, nerves, or other body parts. Moreover, sometimes pain can occur as a result of cancer therapy. Some chemotherapy medicines might induce numbness and tingle in the hands and feet, or, they might create a burning feeling when the drug is injected. Skin inflammation and redness are common side effects of radiotherapy. ${ }^{[9]}$

In clinical practice, pain characteristics and patient factors relevant to cancer pain are commonly utilized to classify or categorize pain into specific domains. ${ }^{[10]}$ The severity of cancer pain is determined by several factors, including the type of cancer, its stage, any other health issues in the patient, and the patient's pain threshold (tolerance of pain). ${ }^{[11]}$ Several pain scales, such as the visual analogue scale, numeric rating scale, and vocal rating scale can be used to reliably evaluate pain severity. In cancer-related pain, the 11-point numeric rating scale, which ranges from 0 (no pain) to 10 (worst pain you can imagine) is the most often used scale. Scores are classified as none (score 0 ), mild (scoring 13 ), moderate (score 4-6), and severe (score 7-10). ${ }^{[12]}$

Palliative care is a specialised type of medical treatment for people residing with a terminal illness. This sort of 
treatment is targeted on alleviating the illness's symptoms as well as the stress and pressure associated with the illness. Palliative care prioritizes the wishes and needs of the affected patient as opposed to their prognosis. ${ }^{[13]}$ Learning to address cancer's signs and symptoms and facet outcomes is a critical factor of dwelling with cancer, and palliative care can assist. ${ }^{[14]}$

\subsection{Medical Cannabis}

The chemicals in medical cannabis are being utilized to treat conditions and symptoms. Even though medical cannabisor medical marijuana and recreational marijuana are the same and are obtained in the same manners, medical cannabis is prescribed for medical purposes. ${ }^{[15]}$ Medical cannabis, acquired from the Cannabis sativa plant, comprises many active compounds with various individual effects on the human body. Nevertheless, only two active compounds are salient for medical utilization. THC (delta-9 tetrahydrocannabinol) found in cannabis is the compound that makes people experience the "high" and CBD (cannabidiol), extracted from hemp plants or manufactured in the laboratory, does not exhibit any effects of 'high' by itself. ${ }^{[16]}$ Medical cannabis is originally derived from 'Sativa' or 'Indica' cultivars. However, as of to the present, there has been an extension in cannabis breeding, yielding various hybrid chemotype strains. Cannabis-based products vary distinctively based on their chemotype strains. ${ }^{[17]}$

\subsubsection{ECS}

The studies of cannabis lead to many discovery advances along with the discovery of ECS- the endocannabinoid system, which is emphasized to be relevant in therapeutic disease treatment. ECS is comprised of endogenous ligands or endogenous cannabinoids (2-arachidonoyl glycerol or 2-AG and arachidonoyl ethanolamide or anandamide), CBR (cannabinoid receptors), and enzymes. This system is a widespread neuromodulatory system, mostly associated with central nervous system (CNS) development, endogenous response, environmental insults, cancer progression, appetite and metabolism, thermogenesis, circadian rhymes, memory, behaviour and mood, pain regulation, heart function, gametogenesis, and synaptic plasticity-changes that occur at the synapses, allowing them to strengthen or weaken in order to communicate. ${ }^{[17-18]}$ ECS has the potential to undergo epigenetic modulation caused by varieties of environmental factors that target the genes encoding for CBRs, then the cannabinoids will influence epigenetic alterations. ${ }^{[17]}$ The production of endocannabinoids stimulates CBR, a class of cell membrane receptors. Two relevant subtypes of CBR have been classified in the endocannabinoid system, CB1 and CB2 receptors. These two CBRs are distinguished by a variety of factors. Their tissue distribution, signalling mechanisms, their agonists (a chemical that binds to the receptors) and antagonists sensitivity, and an amino acid sequence are a few to be considered. The CB1 receptor is prevalent in the brain, controlling the activities and levels of other neurotransmitters and are abounding in GPCR. GPCR, also known as the G-protein coupled receptors, are expressed throughout the gastrointestinal tract and CNS on the axons and axons terminal of neurons. CB1 receptor functions are mostly associated with regulating immediate feedback and adjusting systems. ${ }^{[19]}$ CB2 receptor exists abundantly in the immune tissue with the function of controlling the immune functioning of the body and participating in modulating intestinal inflammation, pain in inflammatory bowel conditions, and contraction. The activation of these two cannabinoids will suppress the production of adenylate cyclase and commence mitogen-activated protein kinase, maximizing intracellular calcium levels. ${ }^{[17]}$

\subsubsection{Phytocannabinoids}

Phytocannabinoids, the chemical constituents of cannabis, are molecules with 
low aqueous solubility and high lipophilic characteristics. The Phytocannabinoids vary differently based on the composition of their fatty compounds. ${ }^{[17]}$ Phytocannabinoids are responsible for affecting ECS through numerous receptors, including CBRs, GPCRs, adrenergic receptors and serotonin receptors. Active phytocannabinoids are classified into subclasses, consisting cannabitrol, cannabichromene, cannabicyclol, cannabielsonin, cannabinodiol, cannabinol, cannabidivarin, cannabidivarin, delta-9-tetrahydrocannabinol, delta-9tetrahydrocannabivarin, cannabigerol and numerous more different subclasses. CBD and THC are the most broadly-researched cannabinoids. One major difference between these two cannabinoids is that THC alters the taker's consciousness but CBD has little to no effect on conscious alteration. CBD is utilized in central nervous system treatment as a result of its therapeutic potential. The patient's pharmacological effects are impacted by CBD through various mechanisms. It works as a negative allosteric modulator (NAM) which binds to the CB1 receptors and decreases the affinity, while also unexpectedly functions as a positive allosteric modulator for CB2 receptors and increases the affinity. ${ }^{[20-21]} \mathrm{CDB}$ has a wide range of therapeutic properties, including anti-inflammatory, anti-oxidant, antitumour, anticonvulsant and neuroprotective effects. CBD also demonstrates its potential as a therapeutic agent to cure obesity, diabetes, ischemia, neurodegenerative diseases and pain control. Excessive amounts of studies have been conducted to determine the effect of CBD on diets. The results have not yet been settled as there are many contradictions among the trial results. One study conducted by Wierucka-Rybak et al. demonstrates that the effects of CBD administered on rats on a high-fat diet increase weight despite the significant reduction in food intake. On the other hand, previous studies have shown the results of the decrease in the bodyweight of rats. THC, another major constituent of Cannabis
Sativa, functions as an agonist of both CB1 and CB2 receptors. THC induces several different physiological processes, including liver and cardiovascular functions, pain perception and modulation of neurotransmitters released in the nervous system. The intervention of CB1 receptors in the CNS, causes THC to operate its psychoactive effect. Some THC pharmacological effect is produced when THC binds to GPR55, 5-HT3A (serotonin receptor subunit) and TRPV 2,3,4 receptors (transient receptor potential channels of vanilloid type 2,3.4). ${ }^{[22]}$

\subsection{Medical Uses of Cannabis on Cancer 1.7.1 Cannabinoids and Cancer Pain}

Cannabis is promising for pain alleviation as there are some patients who do not respond well to some traditional analgesics, and alternative therapeutic possibilities are needed. A small number of studies have shown that higher doses of THC were associated with increased pain relief, but side effects such as drowsiness, vomiting, hypotension, nausea, and mental clouding were still reported. Further researches are still needed since many studies lacked statistical power as a result of an insufficient number of subject. ${ }^{[23]}$

\subsubsection{Cannabis Treating Nausea and Vomiting from Cancer Chemotherapy}

According to 23 randomized controlled trials, a smaller portion of people with cannabinoid medicine experienced nausea and vomiting related to the chemotherapy compared to people with placebo. It was also found that cannabisbased medicine could be as effective as conventional anti-nausea medicines. Even so, there were some side effects of those medicines such as feeling high, dizziness, sedation, and dysphoria, which is feeling uneasy or dissatisfied. ${ }^{[24]}$ There was another study where patients used cannabis over 6 months resulting in a decline in the number of patients with severe pain and the number of opioid use. ${ }^{[25]}$ 


\subsubsection{Cannabis Slowing the Growth of Cancer Cells}

A review in 2019 suggested that substances in cannabis such as CBD and THC could have tumor growth-inhibiting effects on pancreatic cancer, creating a reduction in tumor invasion and growth. Compared with normal tissue, cannabinoid receptors were expressed more in cancer tissues. When combined with gemcitabine, CBD could improve the pancreatic cancer survival rate in some pancreatic cancer models. ${ }^{[26] .}$

\section{Cannabinoids on CIVN}

Post-cancer chemotherapy side effects may include frequent vomiting and anorexia. Prior to the study of cannabinoids, varieties of existing antiemetics had been used to treat these symptoms. However, none of the antiemetics seemed to be successful with gastrointestinal effects. Cannabinoids, including THC and Compazine (prochlorperazine), have been known for their properties to treat and prevent nausea, vomiting, pain, and for stimulating appetite. THC has been confirmed to help treat gastrointestinal effects. This confirmation is supported by the evidence from a study conducted by Sallan SE and colleagues on the antiemetic effect of THC in patients receiving cancer chemotherapy. Both THC and placebos were given to 20 young patients. The result of the study indicates that THC has the potential to treat nausea and vomiting if administered correctly. ${ }^{[27-28]}$

Chemotherapeutic agents and chemotherapeutic metabolites activating neurotransmitter receptors in the gastrointestinal tract and brain result in CIVN (chemotherapy-induced nausea and vomiting). Oral cannabinoids (i.e. dronabinol, nabilone) are used by patients to treat CIVN. Although medical marijuana and oral cannabinoids are both utilized to treat CIVN, medical marijuana has more limitations than oral cannabinoids on cancer. The patient's inability to tolerate smoked marijuana, the risk for atrial fibrillation, myocardial infarct, and chronic bronchitis are these limitations. A majority of clinical studies on antiemetic agents have focused solely on the prevention, rather than the treatment, of CIVN. Nevertheless, in consideration of patients' risk of experiencing CIVN for days after getting their dose of chemotherapy, it is also extensively crucial to identify effective therapies for current CIVN symptoms. ${ }^{\text {[29] }}$

In 1978, oral cannabinoids were approved to be utilized for CIVN treatment. Nonetheless, it is necessary to prescribe CIVN in appropriate amounts for the patients to obviate dizziness, dry mouth, and drowsiness. As of now, oral dronabinol (oral cannabinoids) has been approved to have favourable pharmacokinetics (PK) profile, which is the body's effect on drugs (e.g. absorption, distribution, metabolism, and excretion) and plasma levels of dronabinol. Oral cannabinoids also benefit patients who react refractorily to other antiemetics. ${ }^{[29-30]}$

\section{The Effectiveness of Cannabis on Cancer Patients in Thailand}

The use of Cannabis for medical purposes is legal in Thailand as of 2021 BC. ${ }^{[31]}$ The indication for medical cannabis use is divided into 3 categories. The first category is approved by a study, for example, it is allowed to use cannabis as an antiemetic to stop effects from chemotherapy. The second category is most likely to alleviate the symptom, but more research is needed to support the efficacy and safety. Some examples are palliative care and Alzheimer's disease. As of the third group, medical cannabis should be used under the supervision of a physician, and it may be used as adjuvant therapy or as a last resort. ${ }^{[32]}$

However, research on The Effectiveness of Cannabis used in the Thai Traditional way on Palliative Care Patients in Thailand has been conducted by a number of researchers, using patients at Wat Chom Thong as their subject. Wat Chom Thong is a temple known for taking care of patients with cancer, using cannabis as a 
treatment. With Thailand being a Buddhist country, temples become a shelter, both mental and physical, for the people. Mr. Banthoon Niyamapa or Uncle Tu is the driving force behind the establishment of Wat Chom Thong Dependency Center, which is initiated to help incurably ill individuals who require cannabis treatments. The temple is not a legal hospital but it is a secure haven for everyone, regardless of their gender, age, or religion. The patient's relative must be present at all times when requiring to stay. Patients recuperating from serious illness will be the ones recommended to use Nano Lung Tu's Oil (Cannabis oil) for a variety of purposes, including drip under the tongue, eating, enema, body care, and nutrition. In return, the patient's relatives can cultivate cannabis and learn how to extract the oil for themselves when the patient returns home to recover. The extraction of Cannabis oil by Mr. Banthoon Niyimapa (Lung Tu) follows the procedure of Rick Simpson's extraction (Rick Simpson is a Canadian engineer and a cannabis activist). Despite following the same procedure, Lung Tu uses Sativa, a native cannabis species found in Thailand while Rick Simpson uses Indica. The study discovered from their 98 subjects that 19 distinct forms of cancers from various organs were treated, with liver cancer being the most common (25.5\%). Unfortunately, 12 individuals died out of the 98 who took part in the study. Therefore, $88 \%$ of the subjects who used Nano Lung Tu cannabis oil survived. The researchers concluded that the usage of Thai medical cannabis, which is derived from Thai knowledge, can improve the quality of life for the sufferers. Thichluck Narongvit, who researched on The Effectiveness of Cannabis used in the Thai Traditional way on Palliative Care Patients in Thailand believes that end-of-life patient observations, physical assessments, and enhanced quality of life are thus objective and evidence that should be presented to the government in order to develop Thailand's laws, policies, and public health systems in the future. ${ }^{\text {[3-34] }}$

\section{Effects of Medical Cannabis}

Addiction is one of the possible risks of cannabis use. Research has suggested that 30 percent of marijuana users have a possibility to have a marijuana use disorder. The disorder includes withdrawal symptoms such as mood and sleep difficulties, a decrease in appetite, and cravings. ${ }^{[35]}$ The most often seen effects of marijuana use are the feeling of euphoria, disorientation, anxiety, and paranoia. ${ }^{[36]}$ Research, although with a small number of cases, reported a rare trigger of myocardial infarction. ${ }^{[37]}$ Thus, health effects should be examined closely for the use of medical cannabis on people with a vulnerability to cardiovascular risk factors. Side effects of cannabinoid drugs are related to the dosage and proficiency of the user. Some effects are similar to the effects of marijuana use such as the feeling of euphoria; people with emotional illness, paranoia, or hallucination might find their symptoms worse. Some have reported increased heart rate, decreased blood pressure, dizziness, fainting, hallucinations, dry mouth, and recent memory. ${ }^{[36]}$

There have been researches on marijuana's long-term effects on the brain. The results of research on animals and humans could be slightly conflicting. In animal research, repeated THC exposure could have an adverse effect on the improvement of working memory tasks. ${ }^{[38]}$ A study has also shown that exposure to cannabinoids during the early stages of brain development could lead to irreversible, subtle dysfunction in rat offspring. ${ }^{[39]}$ Some people are also considering the effects of marijuana on IQ levels. Research in humans suggests that the IQ of marijuana users was significantly lower than those who do not use, and quitting the use of marijuana could not recover the lost IQ points. ${ }^{[40]}$ A long-term study among 3385 participants has found that past exposure to marijuana was related to worse verbal memory, but found no association with cognitive functions. ${ }^{[41]}$ 


\section{CASE STUDY}

118 anonymous patients of Siriraj Hospital were asked a series of questions as a part of a study survey conducted by N.Z. in January 2020. This survey focused on the patients' perspective of medical marijuana utilization in Thailand. The responses from the patients disclosed that approximately $20 \%$ out of all the patients reported the use of cannabis for medical purposes. This $20 \%$ is categorized into 3 subgroups. $5 \%$ of all the $20 \%$ are still using medical cannabis, $7.5 \%$ have used once and stopped, and another $7.5 \%$ used for a while and stopped. The reasons given by the $5 \%$ of patients for the continuous utilization of medical cannabis are for curing cancer pain, appetite stimulation, treatment of cancer and sleeping aid. ${ }^{[42]}$

Regardless of the widespread usage of medical cannabis, the majority of patients still lack an understanding of its medical properties. According to the survey, 20\% believe that cannabis can cure cancer, $22 \%$ and $47 \%$, on the other hand, believe that cannabis has the potential to alleviate chemotherapy-induced nausea and vomiting, and alleviate cancer pain. The other $28 \%$ believe that they have a thorough apprehension of medical cannabis. A survey conducted by N.Z on 106 Siriraj Hospital doctors, who have undergone medical cannabis training, suggested that $11 \%$ of the doctors perceived that the patients have an acceptable comprehension of the appropriate usage of medical cannabis. ${ }^{[42]}$

\section{CONCLUSION}

Currently, cannabis is a promising alternative substance for medical purposes. Still, more research is needed on medical cannabis in order to figure out its effects and advantages on humans.THC, CBD and ESC are extracted from the plants and are used for medical purposes. One of its uses is palliative care, a type of medical treatment that focuses on alleviating the illness's symptoms and stress, for cancer patients. Cancer, a disease caused by the uncontrollable growth of cells, can cause pain both by itself and by its treatment. Moreover, there is a study in Thailand on the use of cannabis for cancer treatments. Mr. Banthoon Niyimapa (Lung Tu) follows the procedure of Rick Simpson's extraction in order to extract cannabis oil but changed the species of cannabis to Thailand's native species. Of 98 subjects with cancer, 19 distinct forms of cancers from various organs were treated and 12 individuals died. The result shows that 88 percent of cancer patients treated with cannabis oil survived. Another survey focusing on perspectives of medical marijuana utilization in Thailand resulted in approximately 20 percent out of all the patients reporting the use of cannabis for medical purposes. Additional research on the use of medical cannabis in Thailand is recommended with a larger number of participants and more diverse methods and variables.

\section{Acknowledgement: None}

\section{Conflict of Interest: None}

\section{Source of Funding: None}

\section{REFERENCES}

1. NCI. The Definition of Cancer [Internet]. 2021 [updated 2021 May 5; cited 2021 Oct. 22] Available from https://www.cancer.gov/aboutcancer/understanding/what-is-cancer

2. CancerQuest. How Cancer Spreads (Metastasis) [Internet]. 2021 [updated 2021 Aug. 11; cited 2021 Oct. 22] Available from https://www.cancerquest.org/cancerbiology/metastasis

3. Stanford Health Care. What is Cancer? [Internet]. 2021 [updated 2021 May 5; cited 2021 Oct. 22] Available from https://stanfordhealthcare.org/medicalconditions/cancer/cancer.html

4. Dr Kolin Tidy, Patient.info. Causes of Cancer [Internet]. 2018 [updated 2018 Mar. 1; cited 2021 Oct. 22] Available from https://patient.info/cancer/cancer/causes-ofcancer

5. Cooper GM. The Cell: A Molecular Approach. 2nd edition. Sunderland (MA): Sinauer Associates; 2000. The Development 
and Causes of Cancer. Available from: https://www.ncbi.nlm.nih.gov/books/NBK9 963/

6. Cancer Research. Types of Cancer [Internet]. 2021 [updated 2021 May 4; cited 2021 Oct. 22] Available from https://home.cancerresearch/types-ofcancer/

7. Stanford Health Care. Types of Cancer [Internet]. 2021 [updated 2021 May 5; cited 2021 Oct. 22] Available from https://stanfordhealthcare.org/medicalconditions/cancer/cancer/cancer-types.html

8. Senthil P Kumar. Reporting Characteristics of Cancer Pain: A Systematic Review and Quantitative Analysis of Research Publications in Palliative Care Journals. Indian Journal of Palliative Care. 2011 JanApr; 17(1):57-66.

9. Cancer Research UK. Causes and types of cancer pain [Internet]. 2021 [updated 2021 May 4; cited 2021 Oct. 23] Available from https://www.cancerresearchuk.org/aboutcancer/coping/physically/cancer-and-paincontrol/causes-and-types

10. Caraceni A, Shkodra M. Cancer Pain Assessment and Classification. Cancers (Basel). 2019; 11(4):510.

11. Cancer.org. Facts About Cancer Pain [Internet]. 2019 [updated 2019 Jan. 3; cited 2021 Oct. 23] Available from https://www.cancer.org/treatment/treatments -and-side-effects/physical-sideeffects/pain/facts-about-cancer-pain.html

12. Junya Zhu, Saul N Weingart. Pain intensity in cancer. Annuals of Palliative Medicine. 2012; 1(2):177-178.

13. Getpalliativecare.org. What is Palliative Care? | Definition of Palliative Care | Get Palliative Care [Internet]. 2021 [updated 2021 May 6; cited 2021 Oct. 23] Available from https://getpalliativecare.org/whatis/

14. Michael W. Smith, MD. Palliative Care During Treatment [Internet]. 2021 [updated 2021 Jan. 11; cited 2021 Oct. 23] Available from

https://www.webmd.com/cancer/guide/palli ative-care-cancer-treatment

15. Melinda Ratini, DO, MS. Medical Marijuana FAQ [Internet]. 2020 [updated 2020 Aug. 20; cited 2021 Oct 25] Available from https://www.webmd.com/a-to-zguides/medical-marijuana-faq

16. Peter Grinspoon, MD, Harvard Health. Cannabidiol (CBD)-what we know and what we don't [Internet]. 2021 [updated 2021 Sep. 24; cited 2021 Oct. 25] Available from

https://www.health.harvard.edu/blog/cannab idiol-cbd-what-we-know-and-what-wedont-2018082414476

17. Ladha KS, Ajrawat P, Yang Y, Clarke H. Understanding the Medical Chemistry of the Cannabis Plant is Critical to Guiding Real World Clinical Evidence. Molecules. 2020 Sep 4; 25(18):4042.

18. Lu HC, Mackie K. An Introduction to the Endogenous Cannabinoid System. Biol Psychiatry. 2016; 79(7):516-525.

19. Peter Grinspoon, MD, Harvard Health. The endocannabinoid system: Essential and mysterious [Internet]. 2021 [updated 2021 Aug. 11; cited 2021 Oct. 25] Available from https://www.health.harvard.edu/blog/theendocannabinoid-system-essential-andmysterious-202108112569

20. Tocris. Pharmacological Glossary [Internet]. 2021 [updated 2021 May 5; cited 2021 Oct. 25] Available from https://www.tocris.com/resources/pharmaco logical-glossary

21. Terry P. Kenakin. A Pharmacology Primer. 3rd edn. Amsterdam, Netherlands: Elsevier; 2009. p.129-147

22. Bielawiec P, Harasim-Symbor E and Chabowski A. Phytocannabinoids: Useful Drugs for the Treatment of Obesity? Special Focus on Cannabidiol. Front. Endocrinol. 2020; 11:114.

23. Blake A, Wan BA, Malek L, DeAngelis C, Diaz P, Lao N, Chow E, O’Hearn S. A selective review of medical cannabis in cancer pain management. Ann Palliat Med 2017; 6(Suppl 2):S215-S222.

24. Smith LA, Azariah F, Lavender VT, Stoner NS, Bettiol S. Cannabinoids for nausea and vomiting in adults with cancer receiving chemotherapy. Cochrane Database Syst Rev. 2015; 2015(11):CD009464.

25. Meng H, Dai T, Hanlon JG, Downar J, Alibhai SMH, Clarke H. Cannabis and cannabinoids in cancer pain management. Curr Opin Support Palliat Care. 2020 Jun; 14(2):87-93.

26. Sharafi G, He H, Nikfarjam M. Potential Use of Cannabinoids for the Treatment of Pancreatic Cancer. J Pancreat Cancer. 2019 Jan 25; 5(1):1-7.

27. Ungerleider JT, Andrysiak T, Fairbanks L, Goodnight J, Sarna G, Jamison K. Cannabis 
and cancer chemotherapy: a comparison of oral delta-9-THC and prochlorperazine. Cancer. 1982 Aug 15; 50(4):636-45.

28. Mayo Clinic. Glioblastoma [Internet]. 2020 [updated 2020 Apr. 4; cited 2021 Oct. 30] Available from https://www.mayoclinic.org/diseasesconditions/glioblastoma/cdc-20350148

29. Badowski ME. A review of oral cannabinoids and medical marijuana for the treatment of chemotherapy-induced nausea and vomiting: a focus on pharmacokinetic variability and pharmacodynamics. Cancer Chemother Pharmacol. 2017 Sep; 80(3):441-449.

30. Heller AA, Lockwood SY, Janes TM, Spence DM. Technologies for Measuring Pharmacokinetic Profiles. Annual Review of Analytical Chemistry (Palo Alto Calif). 2018 Jun 12; 11(1):79-100.

31. Narcotics Control Division Cannabis. Law, Announcement, and Regulations [Internet]. 2021 [updated 2021 May 6; cited 2021 Oct. 31] Available from https://cannabis.fda.moph.go.th/principle/

32. Atchariya Fongsri. Medical Cannabis in Thailand [Internet]. 2020 [updated 2020 Jan.; cited 2021 Oct. 31] Available from https://www.skho.moph.go.th/strategy/wpcontent/uploads/2020/01/Cannabis-in-Meduse-Edited-120120.pdf

33. Thichluck Narongvit, Sirikarn Techapichok, Phrakhu Panyawaraphirat, Parinya Jintung, Arisara Maranet. The Effectiveness of Cannabis used in Thai Traditional way on The Palliative Care Patients in Thailand. Journal of the Association of Researchers. 2021; 26(2):219-232.

34. Markham Heid. Rick Simpson Oil (RSO) for Cancer: Does It Work? [Internet]. 2020 [updated 2020 May 17; cited 2021 Oct. 31] Available from https://www.webmd.com/cancer/ricksimpson-oil-for-cancer-overview

35. Hasin DS, Saha TD, Kerridge BT, et al. Prevalence of Marijuana Use Disorders in the United States Between 2001-2002 and 2012-2013. JAMA Psychiatry. 2015; 72(12):1235-1242.
36. MaryAnn De Pietro, CRT, Healthline. Medical Marijuana [Internet]. 2020 [updated 2020 Jul. 21; cited 2021 Nov. 6] Available from https://www.healthline.com/health/medicalmarijuana\#side-effects

37. Mittleman MA, Lewis RA, Maclure M, Sherwood JB, Muller JE. Triggering myocardial infarction by marijuana. Circulation. 2001 Jun 12; 103(23):28052809.

38. Verrico CD, Gu H, Peterson ML, Sampson AR, Lewis DA. Repeated $\Delta 9$ tetrahydrocannabinol exposure in adolescent monkeys: persistent effects selective for spatial working memory. Am J Psychiatry. 2014; 171(4):416-425.

39. Campolongo P, Trezza V, Cassano T, et al. Perinatal exposure to delta-9tetrahydrocannabinol causes enduring cognitive deficits associated with alteration of cortical gene expression and neurotransmission in rats. Addict Biol. 2007; 12(3-4):485-495.

40. Filbey FM, Aslan S, Calhoun VD, et al. Long-term effects of marijuana use on the brain. Proc Natl Acad Sci U S A. 2014; 111(47):16913-16918.

41. Auer R, Vittinghoff E, Yaffe K, Künzi A, Kertesz SG, Levine DA, Albanese E, Whitmer RA, Jacobs DR Jr, Sidney S, Glymour MM, Pletcher MJ. Association Between Lifetime Marijuana Use and Cognitive Function in Middle Age: The Coronary Artery Risk Development in Young Adults (CARDIA) Study. JAMA Intern Med. 2016 Mar;176(3):352-61.

42. Zinboonyahgoon N, Srisuma S, Limsawart W, Rice ASC, Suthisisang C. Medicinal cannabis in Thailand: 1-year experience after legalization. Pain. 2021 Jul 1; 162(Suppl 1):S105-S109.

How to cite this article: Ekarattanawong S, Ketphan V, Rojcharoenchai Y. The use of medical cannabis on cancer in Thailand. International Journal of Science \& Healthcare Research. 2021; 6(4): 252-260. DOI: https:// doi.org/10.52403/ijshr.20211036 